\title{
Experiencia quirúrgica en el manejo de litiasis urinaria en la población pediátrica: estudio multicéntrico
}

\section{Surgical Experience in Management of Urinary Lithiasis in Pediatric Population: Multicentric Study}

\author{
Victor H. Figueroa ${ }^{1}$ Lynda Torres ${ }^{2}$ Carlos Felipe García ${ }^{3}$ Stefania Prada ${ }^{2}$ Mariangel Castillo 4 \\ Jaime Francisco Pérez ${ }^{5}$
}
${ }^{1}$ Urólogo Pediatra, Unidad de Urología, Clínica Carlos Ardila Lulle y Facultad de Medicina, Universidad Autónoma de Bucaramanga, Bucaramanga, Colombia
2 Médico Residente de Urología, Unidad de Urología, Hospital Universitario San Ignacio y Facultad de Medicina, Pontificia Universidad Javeriana, Bogotá D.C., Colombia
${ }^{3}$ Médico Residente de Urología, Unidad de Urología, Clínica Carlos Ardila Lule y Facultad de Medicina, Universidad Autónoma de Bucaramanga, Bucaramanga, Colombia
${ }^{4}$ Nefróloga Pediatra, Hospital Universitario San Ignacio, Bogotá D.C., Colombia
${ }^{5}$ Urólogo Pediatra, Unidad de Urología, Hospital Universitario San Ignacio y Facultad de Medicina, Pontificia Universidad Javeriana, Bogotá D.C., Colombia

Urol Colomb 2018;27:277-281.

\author{
Address for correspondence Dra. Lynda Torres, MD, Pontificia \\ Universidad Javeriana, Carrera 7 No. 40-62, Bogotá, Colombia \\ 110231 (e-mail: lynda.torresc@gmail.com).
}

\section{Resumen}

Palabras Clave

- litiasis urinaria

- urología pediátrica

- manejo quirúrgico

- ureterolitotomía endoscópica retrógrada

- cirugía abierta

- nefrolitotomía percutánea
Introducción y Objetivos La incidencia de litiasis urinaria en pediatría ha venido en aumento en los últimos años. Las indicaciones quirúrgicas están bien establecidas en la literatura, y con la disponibilidad actual de instrumental quirúrgico de calibre reducido diseñado para pacientes pediátricos, el manejo de esa entidad, ha tenido una evolución dramática. Mediante este estudio, se describe la experiencia quirúrgica de dos centros de referencia en el manejo de litiasis urinaria en población pediátrica.

Métodos se realizó un estudio multicéntrico, observacional descriptivo y retrospectivo. Se incluyeron pacientes menores de 18 años que fueron sometidos a algún manejo quirúrgico para enfermedad litiásica urinaria entre diciembre de 2010 y marzo de 2017. Los datos demográficos, evaluación pre-quirúrgica, características del cálculo, complicaciones y cálculos residuales fueron registrados, así como la tasa de éxito de los procedimientos. Se realizó un análisis estadístico descriptivo con medidas de frecuencia utilizando el software SPSS.

Resultados Un total de 18 pacientes y 19 procedimientos fueron incluidos. La media de edad fue 7,9 años (1-16), las presentaciones clínicas más comunes fueron dolor lumbar e infección de vías urinarias en un $42 \%$ y un $37 \%$ respectivamente. El diagnóstico se realizó mediante Urotac en la mayoría de pacientes (83\%). La media del número y tamaño de los cálculos fue 1,9 (DE 3,1) y 14,8 mm (DE 10,92) respectivamente. La received

October 27, 2017

accepted

April 24, 2018

published online

October 4, 2018
DOI https://doi.org/

10.1055/s-0038-1656543.

ISSN 0120-789X.

eISSN 2027-0119.
Copyright ( 2 2018, Sociedad Colombiana License terms de Urología. Publicado por Thieme Revinter Publicações Ltda., Rio de Janeiro, Brazil. Todos los derechos reservados. 
localización más común fue piélica (31\%), seguido de uréter distal (26\%), 2 (10\%) pacientes tuvieron cistolitiasis y 4 (21\%) pacientes tuvieron cálculos en diferentes localizaciones. Se realizaron 7 (37\%) ureterolitotomías retrógradas endoscópicas (ULE) semirrígidas, 6 (31\%) ULE flexibles, 3 (16\%) cistolitotomías y 2 (10\%) nefrolitotomías percutáneas. Un $53 \%$ de los casos, tenían catéter doble J previo al procedimiento y en un $68 \%$ de los casos, se dejó catéter doble J después del procedimiento. La tasa de éxito de las cirugías fue de un $89 \%$. No se documentaron complicaciones intraoperatorias ni postoperatorias.

Conclusiones La litiasis urinaria en la población pediátrica es una entidad poco común. El tratamiento quirúrgico tiene alta tasa de éxito y es seguro. Se necesitan estudios con mayor nivel de evidencia para determinar el esquema terapéutico quirúrgico apropiado de esa población.

\section{Abtract}

\section{Keywords}

- urolithiasis

- pediatric urology

- surgical management

- ureteroscopic management

- open surgery

- percutaneous nephrolythotomy
Introduction and objectives Urolithiasis incidence in pediatric population has been increasing in the last few years. Surgical indications are well established in literature, and due to the current availability of surgical instruments of decreased caliber designed for pediatric patients its management has had a dramatic evolution. With this study, we describe the surgical experience of two referral centers in management of urinary lithiasis in pediatric population.

Methods A multicentric, retrospective, observational and descriptive study was performed. We included patients under 18 who were treated for urolithiasis in a surgical manner between december 2010 and march 2017. The demographic data, presurgical evaluation, stone characteristics, complications and the presence of residual calculi was registered, as well as the success rate of each procedure. We performed a descriptive statistical analysis with frequency measures using the SPSS software.

Results A total of 18 patients and 19 procedures were included. The mean age was 7.9 years (1-16), the most common clinical presentations were lumbar pain and urinary tract infection in a $42 \%$ and $37 \%$ respectively. The diagnosis was made through an CT scan in most patients $(83 \%)$. The mean number and size of stones was $1,9($ ED3 11$)$ and $14,8 \mathrm{~mm}$ (ED 10,92) respectively. The most common localization was pielic (31\%), then distal uréter (26\%), bladder (10\%) and multiple localizations (21\%). Seven semirrigid URS (37\%), six flexible URS (16\%), three cistolythotomies (16\%) and two percutaneous nephrolythotomies (10\%) were performed. In $53 \%$ of the cases the patient had a JJ catheter before the procedure and in $68 \%$ of the cases a JJ catheter was placed after the procedure. The success rate was $89 \%$. No intraoperative or postoperative complications were documented.

Conclusions The urinary lithiasis in the pediatric population is a very infrequent entity. Surgical management has a high success rate and is safe. More studies are needed with a higher evidence level to determine the apropiate surgical therapeutic regimen in this population.

\section{Introducción}

La incidencia de la enfermedad litiásica ha aumentado globalmente en los últimos 25 años. A pesar de que la incidencia de litiasis es baja en la población pediátrica, ${ }^{1}$ ese aumento no ha sido ajeno a esa población. Se ha visto un incremento importante en su incidencia y prevalencia en los últimos años, alcanzado en países como Estados Unidos de 18,5 por cada 100.000 habitantes. $^{2}$ La edad de presentación se encuentra entre los 7 y los 8 años de edad. ${ }^{3,4}$ Al analizar el género como factor de riesgo se encuentra que los niños tienen una tasa mayor de nefrolitiasis durante la primera década de la vida, mientras que en la segunda década las mujeres presentan un riesgo más alto por razones aún no conocidas. La presentación clínica es variable, encontrando el dolor abdominal como el síntoma más común (55\%-70\%), seguido por hematuria macroscópica (14\%-33\%) e infección de vías urinarias $(8 \%-46 \%) .{ }^{5-7}$ De manera similar a los adultos, la 
expulsión espontanea de los litos depende del tamaño y la localización. ${ }^{7,8}$ Con el incremento en la incidencia de la enfermedad litiásica en niños se ha visto a su vez un aumento en el uso de imágenes con radiación ionizante, como la urografía excretora y tomografía computarizada (Urotac). Estudios recientes recomiendan a la ultrasonografía como el estudio de elección siendo un método seguro, sin radiación ionizante y buen desempeño diagnóstico. ${ }^{9}$ Quizás los cambios más dramáticos se han visto en el manejo quirúrgico de la litiasis en pediatría. Actualmente hay indicaciones establecidas de acuerdo a guías desarrolladas por sociedades internacionales en el tratamiento de los cálculos en niños y adolescentes ${ }^{10}$ y con el rápido desarrollo y disponibilidad de instrumental quirúrgico de calibre reducido diseñado para la población pediátrica, el manejo de esa entidad, ha tenido una evolución dramática. ${ }^{11}$

El objetivo de este estudio, es describir mediante una serie de casos, la experiencia quirúrgica de dos centros de referencia en el manejo de litiasis urinaria en la población pediátrica.

\section{Materiales y Métodos}

Se realizó la búsqueda retrospectiva de historias clínicas de dos centros de referencia desde diciembre de 2010 hasta marzo de 2017. Se incluyeron todos los pacientes menores de 18 años sometidos a alguna intervención quirúrgica para el manejo de litiasis de la vía urinaria. En todos los casos el diagnostico de litiasis fue realizado por medio de imágenes de las vías urinarias. La decisión del abordaje quirúrgico fue basado en la localización y tamaño del cálculo. Para la recolección de los datos se tuvieron en cuenta las características demográficas, pre-quirúrgicas, características de los cálculos, efecto obstructivo, tipo de cirugía, complicaciones y cálculo residual. El éxito de la cirugía se definió como la tasa libre de cálculos, siendo esa un cálculo residual menor de $2 \mathrm{~mm}$. Los datos fueron analizados en el software SPSS versión 17. Las variables continuas se expresaron como promedios con desviaciones estándar (DE). Las pruebas de $t$-test no pareada fueron usadas para comparar variables continuas. Un valor de $p$ igual o menor de 0,05 se consideró estadísticamente significativo.

\section{Resultados}

Se incluyeron en total 18 pacientes, en los que se realizaron 19 procedimientos quirúrgicos. La edad promedio al procedimiento quirúrgico fue de 7,9 años (mediana 4,3; rango 1-16), de ellos 12 (66\%) pacientes, eran mujeres y 6 (33\%) hombres. Cuando se analizó por centro quirúrgico, se encontró una edad menor en los pacientes de la región de Santander con una media de edad de 7,1 años (mediana 4,65; rango 1-16), comparado con Bogotá de 9,8 años (mediana 9; rango $1-16) p=0,2187$. Con respecto al género, las mujeres tenían una edad promedio mayor que los hombres con 9,2 años (media 10; rango 1-16) versus 3,9 años (media 3,45; rango 1-9) $p=0,030$. La presentación clínica más comúnmente encontrada fue dolor abdominal en 8 pacientes, seguido de infección de vías urinarias en 7 , hematuria macroscópica en
4 y litiasis como hallazgo incidental en 2. En 2 pacientes se encontró más de un síntoma.

A 15 pacientes se les realizó Urotac, en todos los casos ese examen fue solicitado previo a la remisión con urología pediátrica. En dos de los 18 casos estudiados, se encontró patología bilateral. El promedio de cálculos y tamaño fue 1,9 (DE 3,21; rango 1-15) y $14,8 \mathrm{~mm}$ (DE 10,92 ; rango $3-30$ ) respectivamente. En 12 casos se encontraron cambios por efecto obstructivo.

La localización de los cálculos en los 18 pacientes se dio de la siguiente manera: 6 en la pelvis renal, 5 en uréter distal, 1 uréter proximal, 1 sistema calicial inferior, 2 vesical y 4 tuvieron cálculos en diferentes localizaciones ( $\mathbf{- T a b l a ~ 1 ) . ~}$

Si subdividimos pacientes por localización, encontramos una edad inferior en aquellos casos con litiasis renal comparado con los que presentaron cálculos ureterales y vesicales, 5,5 años (mediana 2,6; rango 12-192) y 12,3 años (mediana 10; rango 12-192) respectivamente.

Respecto a los procedimientos; de forma endoscópica se realizaron: 2 nefrolitotomías percutáneas, 7 ULE semirrígida, 6 ULE flexible y 2 cistolitotomías (en uno de los pacientes el cálculo ureteral migró a la vejiga), adicionalmente, se hicieron 2 procedimientos abiertos (pielolitotomía y cistolitotomía). Tres pacientes tuvieron múltiples intervenciones. El intervalo de tiempo entre cada intervención fue entre 2 y 60 meses. De los anteriores, a un paciente se le realizó cirugía bilateral.

De los 11 casos en los que se realizó ULE (semirrígida o flexible), el catéter doble J fue usado en 7 pacientes con el fin de dilatar el uréter y facilitar el abordaje endoscópico (66,3\%), con un promedio de edad de 5,2 años (mediana 2,$6 ; 1-10$ ) comparado con 12,2 años (mediana $15 ; 12-16$ ) en aquellos en los que no se derivaron previo a la ULE, $p=0,032$. Solo en un

Tabla 1 Características de la población incluída

\begin{tabular}{|l|l|}
\hline Pacientes & 18 \\
\hline Edad (promedio) & 95 meses (DE 68) \\
\hline Mujer: hombre & $13: 6$ \\
\hline Edad hombres & 47 meses (DE 46,8) \\
\hline Edad mujeres & 111 meses (DE 71,2) \\
\hline Presentación & $8(42 \%)$ \\
\hline Dolor abdominal & $7(37 \%)$ \\
\hline Infección de vías urinarias & $4(21 \%)$ \\
\hline Hematuria macroscópica & $2(10 \%)$ \\
\hline Asintomático/incidental & $1(5 \%)$ \\
\hline Localización del cálculo & $6(31 \%)$ \\
\hline Calicial & $1(5 \%)$ \\
\hline Piélico & $5(26 \%)$ \\
\hline Ureteral superior & $2(10 \%)$ \\
\hline Ureteral distal & $4(21 \%)$ \\
\hline Vesical & \\
\hline Múltiple localización* &
\end{tabular}

*Un paciente con cálculo coraliforme completo izquierdo, un paciente con múltiples cálculos en todos los cálices y piélicos 
Tabla 2 Resultados

\begin{tabular}{|l|l|}
\hline Cirugía múltiple & $3(16 \%)$ \\
\hline Tipo de cirugía & \\
\hline Nefrolitotomía percutánea & $2(10 \%)$ \\
\hline ULE semirígida & $7(37 \%)$ \\
\hline ULE flexible & $6(31 \%)$ \\
\hline Cistolitotomía endoscópica & $2(10 \%)$ \\
\hline Pielolitotomía abierta & $1(5 \%)$ \\
\hline \multicolumn{1}{|c|}{ Cistolitotomía abierta } & $1(5 \%)$ \\
\hline Catéter doble J Pre quirúrgico & $9(53 \%)$ \\
\hline Catéter doble J Post quirúrgico & $11(68 \%)$ \\
\hline Nefrostomía & $2(10 \%)$ \\
\hline Tamaño cálculo residual & 4 y 5 mm \\
\hline
\end{tabular}

Abreviatura: ULE, Ureterolitotomía Retrógrada Endoscópica.

paciente no se realizó derivación con catéter doble J después del procedimiento endoscópico ureteral (edad de 15 años). Derivación con nefrostomía fue usada en 2 pacientes posterior a la realización de nefrolitotomía percutánea. En solo un paciente de 10 años se utilizó camisa ureteral durante la realización de ULE flexible.

No se documentaron complicaciones postoperatorias en ninguno de los 19 casos. La tasa de éxito de la cirugía fue de un $89 \%$. En 9 pacientes se confirmó con imágenes. De los 2 pacientes con cálculo residual el tamaño fue de 4 y $5 \mathrm{~mm}$ (-Tabla 2).

\section{Discusión}

La presencia de litiasis urinaria en la edad pediátrica es infrecuente y la experiencia en el manejo quirúrgico limitada. Con la disponibilidad de instrumental quirúrgico de calibre reducido diseñado para esa población, el manejo de esa patología ha tenido grandes avances en los últimos años.

Abhishek y col., en India documentaron la presencia de cálculos de la vía urinaria con predominio en el género masculino con una tasa de $3: 1 .^{7}$ Sin embargo, esos resultados no son comparables con otros resultados o con las características de la población adulta. De hecho, en el estudio de Pietrow y col., desarrollado en Nashville Tennessee $^{12}$ la presencia de cálculos fue igual en ambos géneros. En nuestro estudio encontramos que las mujeres tienen una incidencia más alta con una edad media de 7,9 años que corresponde a la etapa prepuberal, y analizando la media de edad diferencial según el género, se observa una presentación más temprana en hombres (3,9 años) que en mujeres (9,2 años). La relación hombre: mujer en nuestro estudio fue $1: 2$. Hallazgos similares fueron vistos por Sas y col., ${ }^{13}$ quien describió que en las mujeres la aparición de cálculos en edad pediátrica se da justo después de la pubertad, lo cual puede estar asociado a las hormonas sexuales mientras que en los hombres se da en una edad más temprana.
De forma interesante nuestros hallazgos sugieren que los pacientes de menor edad tienen una incidencia más alta de nefrolitiasis, encontrando una distribución similar en los adultos en los casos de mayor edad. Una distribución parecida fue encontrada en los estudios de Pietrow y col., ${ }^{12}$ y posteriormente de Abhishek ${ }^{7}$ quienes sugieren que la localización más frecuente de cálculos es a nivel renal. En términos de manifestación clínica, según el estudio de Abhishek y col., la presentación clínica más frecuente fue el dolor abdominal lo cual corresponde con nuestro estudio en el $42 \%$ de los casos. ${ }^{7}$

En cuanto al manejo quirúrgico de la litiasis en edad pediátrica, se ha visto a través de varios estudios que es una opción eficaz. En el estudio de Ishii y col., ${ }^{14}$ realizado en el Reino Unido, se demostró una tasa media libre de cálculos del $85,5 \%$. En nuestro estudio, la tasa de éxito fue similar, siendo de un $89 \%$. En total, los procedimientos más realizados fueron por vía ureteroscópica con un 37\% de ellos manejados con ULE semirrígida y un 31\% con ULE flexible. Chedgy y col., ${ }^{15}$ manejaron 21 pacientes por vía ureteroscópica para cálculos en riñón y uréter con falla en solo el 5\% de los pacientes. Demostraron que los resultados no varían con la edad, lo cual es concordante con los resultados de Dangle y col., ${ }^{16}$ quienes no encontraron diferencia tanto en resultados como en complicaciones en pacientes prepuberales versus adolescentes sometidos a manejo ureteroscópico. Una herramienta que encontramos de utilidad en nuestra cohorte fue la derivación urinaria con catéter doble J en pacientes en quienes no se logró el paso del equipo endoscópico de forma primaria. Realizando ese paso, logramos una adecuada dilatación ureteral y el paso de instrumental sin dificultad logrando acceder a la pelvis renal y cálices en el $100 \%$ de los casos.

En términos de complicaciones del manejo ureteroscópico, Chedgy y col., reportaron como complicación más frecuente la persistencia de dolor en el flanco, la cual estuvo presente en el $17 \%$ de sus pacientes. En nuestra población, no se encontraron complicaciones. ${ }^{15}$ Hay que resaltar el uso de catéter doble J prequirúrgico en el $58 \%$ de los pacientes llevados a procedimientos ureteroscópicos, lo cual consideramos tuvo una repercusión importante en la nula tasa de complicaciones postquirúrgicas. Esa conducta se encuentra soportada por varios autores, y se recomienda especialmente en pacientes prepuberales con uréteres de bajo calibre ${ }^{17}$

La nefrolitotomía percutánea también juega un papel fundamental en el manejo de litiasis en pacientes pediátricos. De hecho, se considera el patrón de oro en cálculos mayores a $2 \mathrm{~cm}$. En un estudio realizado en Beijing $^{18}$ en el que 56 pacientes fueron sometidos a nefrolitotomía percutánea, se obtuvo una tasa libre de cálculos al egreso de un $94 \%$ con un tiempo promedio de hospitalización de 7,1 días y complicaciones dadas por fiebre en 5 pacientes y derrame pleural en 1 paciente, demostrando que se trata de un procedimiento con alta tasa de éxito y exento de complicaciones. En nuestra población de estudio, 2 pacientes fueron manejados con nefrolitotomía percutánea que corresponde al $10 \%$ de los pacientes analizados. 
Nuestra investigación presenta limitaciones por el tipo de estudio y el bajo número de pacientes incluidos por ser una entidad poco frecuente. Adicionalmente, los datos son retrospectivos existiendo riesgo de sesgo en cuanto a la selección, recolección de la información y además puede carecer de validez externa. No obstante, en nuestro conocimiento, es el primer informe acerca del manejo quirúrgico de la litiasis en pediatría en Colombia, por lo tanto, los datos obtenidos aportan información epidemiológica y clínica de la enfermedad litiásica, mostrando que el manejo quirúrgico tiene alta tasa de éxito.

\section{Conclusión}

El tratamiento quirúrgico de la enfermedad litiásica pediátrica, es una alternativa terapéutica segura con una alta tasa de éxito. En pacientes pequeños en quienes no es posible el paso del equipo endoscópico, se recomienda considerar realizar derivación urinaria temporal para dilatar el uréter y permitir la realización de la cirugía endoscopio ureteral. Se necesitan estudios con mayor nivel de evidencia para determinar el esquema terapéutico quirúrgico apropiado de esa población.

\section{Responsabilidades éticas}

Protección de personas y animales. Los autores declaran que para esta investigación, no se han realizado experimentos en seres humanos ni en animales.

Confidencialidad de los datos. Los autores declaran que han seguido los protocolos de su centro de trabajo sobre la publicación de datos de pacientes.

Derecho a la privacidad y consentimiento informado. Los autores declaran que en este artículo no aparecen datos de pacientes.

\section{Financiación}

No existe una fuente de financiación para este trabajo.

Conflicto de Intereses

No se reportan conflictos de intereses.

\section{Bibliografía}

1 García HA, Benavidez Solarte P, Posada España P. Fisiopatología asociada a la formación de cálculos en la vía urinaria. Urol Colomb 2016;25(02):109-117
2 Dwyer ME, Krambeck AE, Bergstralh EJ, Milliner DS, Lieske JC, Rule AD. Temporal trends in incidence of kidney stones among children: a 25-year population based study. J Urol 2012;188 (01):247-252

3 Jørgensen L, Jacobsen BK, Wilsgaard T, Magnus JH. Walking after stroke: does it matter? Changes in bone mineral density within the first 12 months after stroke. A longitudinal study. Osteoporos Int 2000;11(05):381-387

4 Erbagci A, Erbagci AB, Yilmaz M, et al. Pediatric urolithiasisevaluation of risk factors in 95 children. Scand J Urol Nephrol 2003;37(02):129-133

5 Milliner DS, Murphy ME. Urolithiasis in pediatric patients. Mayo Clin Proc 1993;68(03):241-248

6 Sternberg K, Greenfield SP, Williot P, Wan J. Pediatric stone disease: an evolving experience. J Urol 2005;174(4 Pt 2):1711-1714, discussion 1714

7 Abhishek KJ, Kumar J, Mandhani A, Srivastava A, Kapoor R, Ansari MS. Pediatric urolithiasis: experience from a tertiary referral center. J Pediatr Urol 2013;9(6 Pt A):825-830

8 Van Savage J, Palanaca L, Anderson R, Rao G. Slaughenhoupt. Treatment of distal ureteral stones in children: similarities to AUA guidelines in adults. J Urol 2000;164(3 Pt 2):1089-1093

9 Türk C, Knoll T, Petrik A, et al. Guidelines on Urolithiasis. Eur Urol 2015:1-71

10 Assimos D, Krambeck A, Miller NL, et al. Surgical Management of Stones: American Urological Association/Endourological Society Guideline, PART I. J Urol 2016;196(04):1153-1160

11 Long CJ, Srinivasan AK. Percutaneous nephrolithotomy and ureteroscopy in children: evolutions. Urol Clin North Am 2015; 42(01):1-17

12 Pietrow PK, Pope JC IV, Adams MC, Shyr Y, Brock JW III. Clinical outcome of pediatric stone disease. J Urol 2002;167(2 Pt 1):670-673

13 Sas DJ, Becton LJ, Tutman J, Lindsay LA, Wahlquist AH. Clinical, demographic, and laboratory characteristics of children with nephrolithiasis. Urolithiasis 2016;44(03):241-246

14 Ishii H, Griffin S, Somani BK. Flexible ureteroscopy and lasertripsy (FURSL) for paediatric renal calculi: results from a systematic review. J Pediatr Urol 2014;10(06):1020-1025. Doi: 10.1016/j. jpurol.2014.08.003

15 Chedgy ECP, Griffin SJ, Dyer JP, Somani BK. Ureteroscopy for Paediatric Renal Tract Stones - Outcomes from a Tertiary European Centre. Urol Int 2015;95(03):320-323

16 Dangle PP, Bandari J, Lyon TD, et al. Outcomes of Ureteroscopic Management of Pediatric Urolithiasis: A Comparative Analysis of Prepubertal and Adolescent Patients. Urology 2016; 89:103-106

17 Reddy PP, Defoor WR. Ureteroscopy: The standard of care in the management of upper tract urolithiasis in children. Indian J Urol 2010;26(04):555-563

18 Xiao B, Zhang X, Hu WG, et al. Mini-percutaneous nephrolithotomy under total ultrasonography in patients aged less than 3 years: A single-center initial experience from China. Chin Med J (Engl) 2015; 128(12):1596-1600 